\title{
Cancer Genetics Network
}

National Cancer Institute

\section{Source}

National Cancer Institute. Cancer Genetics Network. NCI Thesaurus. Code C19256.

A network dedicated to studying inherited predispositions to cancer. Includes 8 centers and an informatics and information technology group. It will integrate discoveries into medical applications and address psychosocial, ethical, legal and other implications related to inherited genetic susceptibility. 